\title{
The Phase Only Transform for unsupervised surface defect detection
}

\author{
Dror Aiger Hugues Talbot \\ Université Paris-Est, Laboratoire d'Informatique Gaspard-Monge, Equipe A3SI \\ ESIEE Paris, 2 Boulevard Blaise-Pascal, 93162 Noisy-le-Grand Cedex France \\ \{d.aiger,h.talbot\}@esiee.fr
}

\begin{abstract}
We present a simple, fast, and effective method to detect defects on textured surfaces. Our method is unsupervised and contains no learning stage or information on the texture being inspected. The new method is based on the Phase Only Transform (PHOT) which correspond to the Discrete Fourier Transform (DFT), normalized by the magnitude. The PHOT removes any regularities, at arbitrary scales, from the image while preserving only irregular patterns considered to represent defects. The localization is obtained by the inverse transform followed by adaptive thresholding using a simple standard statistical method. The main computational requirement is thus to apply the DFT on the input image. The new method is also easy to implement in a few lines of code. Despite its simplicity, the methods is shown to be effective and generic as tested on various inputs, requiring only one parameter for sensitivity. We provide theoretical justification based on a simple model and show results on various kinds of patterns. We also discuss some limitations.
\end{abstract}

\section{Introduction}

Vision-based inspection of surfaces has many real-world applications, for instance industrial wood, steel, ceramic and silicon wafers, fruits, aircraft surfaces and many more. It is in high demand in industry in order to replace the subjective and repetitive process of manual inspection. A comprehensive survey on recent developments in vision based surface inspection using image processing techniques, particularly those that are based on texture analysis methods, can be found in [21]. According to this work, one can divide the methods for surface defect detection into four categories, namely: statistical approaches, structural approaches, filter-based methods, and model-based approaches. A significant differentiating factor in visual inspection approaches is that of supervised classification versus novelty detection. For applications where both normal and defective samples can be easily obtained, supervised classification based approaches are usually favored. However, when defects are unpredictable and defective samples are unavailable, novelty detection is more desirable.

Texture is one of the most important characteristics in identifying defects or flaws. Much effort were invested in extracting useful texture features [7, 18, 20]. Statistical texture analysis methods investigate the spatial distribution of pixel values. In structural approaches, texture is characterized by primitives or texture elements, and the spatial arrangement of these primitives [16]. The goals of structural approaches are to extract texture primitives, and to model the spatial arrangement. Filter based approaches share a common characteristic, which is applying filter banks on the image and compute the energy of the filter responses. These methods can be divided into spatial domain, frequency domain, and joint spatial/spatial-frequency domain techniques. Model based methods include, among many others, fractal models [6], autoregressive models [4], random field models [10], and the texem model [22].

In a novelty detection task, the task is to identify whether an input pattern is an expected part of the data or unknown. As for defect detection, it involves assigning a normal or abnormal label to a pattern (e.g. a surface or a pixel). In contrast to supervised classification, novelty detection only needs the normal samples for training purposes and usually uses a distance measure and a threshold for decision making. Recently, Markou and Singh [14, 15] gave a detailed review of novelty detection approaches, using statistical and neural network based approaches. Statistical parametric approaches are commonly used in visual inspection $[12,9,1,13]$. A fundamental assumption is that the data distribution is Gaussian in nature, thus, it can be easily statistically modeled by means and covariances.

Working in the frequency domain is closely related to our contribution. Many methods apply filtering in the frequency domain, particularly when no straightforward kernel can be found in the spatial domain. The image is transformed into the Fourier domain, multiplied with the filter function and then re-transformed into the spatial domain. In [3], Coggins and Jain used ring filters and orientation fil- 
ters for feature extraction. DAstous and Jernigan [5] used peak features, such as strength and area, and power distribution features, such as power spectrum eigenvalues and circularity, to discriminate textures. In [19], the authors used the Fourier transform (FT) to reconstruct textile images for defect detection. Chan and Pang [2] extracted harmonic peaks from horizontal and vertical power spectrum slices. The phase of the DFT was used for matching images by the Phase Only Correlation (POC) [8]. It was also used for image coding [11].

The main focus of this paper is to develop an unsupervised method for defect detection. We concentrated on a method that does not require any prior information or learning stage. This solves the practical problem of collecting good examples of good patterns and defected ones. In addition, in many inspection applications, the pattern of the inspected surface is not known a-priori. As we work in the frequency domain, we have the advantages of a global view, solving the problems of selecting good kernel sizes. On the other hand, our method localizes defects without the need for any post processing. The idea is very simple. Instead of trying to detect peaks in Fourier transform of the input images, it simply removes all regularities in the image of various sizes and patterns at once by normalizing the FT of the input image by its magnitude. Through this operation, only the phase information remains while all regular patterns at all scales are removed. The localization of the defects is achieved by transforming back to the spatial domain. We show that since no analysis is being performed in the Fourier domain, removing regularities by normalizing the magnitude serves as a multiscale regularity removal, therefore, random textures are effectively removed as well.

\section{The importance of the phase}

In their important paper, Oppenheim and Lim investigated the important of phase in signals [17]. We review here some of their insights. In the Fourier representation of signals, spectral magnitude and phase tend to play different roles and in some situations, many of the important features of a signal are preserved even if only the phase is retained. This is not true in general for the spectral magnitude. This observation about phase has been made in a number of different contexts and applications. In general, reconstructing an object from the magnitude only is not of much value in representing the original object, whereas reconstructions from the phase only have many important features in common with the original objects. A phase-only image has Fourier transform phase equal to that of the original image and a FT magnitude of unity. Figure 1 show reconstruction of Lenna with magnitude only and phase only. It shows that although the rebuilt image contains the same frequency terms as the original input, the magnitude-only transform bears no resemblance to the original image, it
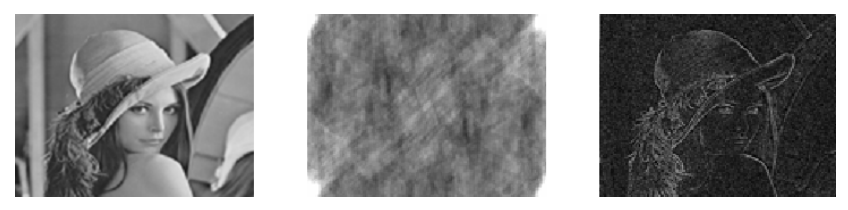

Figure 1. The Phase only inverse Vs. Magnitude Only inverse left: original, middle: magnitude only, right: phase only

does not allow recognition due to the missing phase. Conversely, a phase-only reconstruction shows a picture similar to the shape of the original Lenna. It appears that the phase contains significant information, especially regarding the edge location. In addition, the phase only transform removes periodicity and regularity so it does more than just preserving the edges. In Section 3.2 we model the problem, give some theoretical justifications and explain why it works.

\section{The new method}

\subsection{Applying the Phase Only Transform}

In the context of this paper, our goal is not to reconstruct a signal from its FT. In fact, we wish to do almost the opposite, namely, to filter out most patterns. We wish to eliminate part of the signal while preserving other. The above discussion provides a way to do it for our application of defect detection in images. Our purpose is to eliminate the so-called regular patterns while preserving correspondingly "rare" events in the image which can be considered to be defects. The fact that phase-only reconstruction preserves much of the correlation between signals would suggest that the location of events tends to be preserved. If we assume that in our application, a non defective region contains non localized structures (e.g. regular patterns or homogeneous regions) and that a defect is well localized, it is reasonable to use the phase to filter all non localized patterns (see Section 3.2).

Our algorithm is very simple and can be implemented in a few lines of code. The first step is to apply the DFT on the input (real) image. The discrete two-dimensional Fourier transform of an image array $F(u, v)$ is defined in series form as:

$$
\mathcal{F}(u, v)=\frac{1}{N} \sum_{j=0}^{N-1} \sum_{k=0}^{N-1} F(j, k) \exp \left\{\frac{-2 \pi i}{N}(u j+v k)\right\}
$$

where $i=\sqrt{-1}$. The indices $(u, v)$ are called the spatial frequencies of the transformation. The result is a matrix of complex numbers in the frequency domain,

$$
\mathcal{F}(u, v)=\mathcal{R}(u, v)+i \mathcal{I}(u, v)
$$


or in magnitude and phase-angle form,

$$
\mathcal{F}(u, v)=\mathcal{M}(u, v) \exp \{i \Phi(u, v)\}
$$

where

$$
\mathcal{M}(u, v)=\sqrt{\mathcal{R}^{2}(u, v)+\mathcal{I}^{2}(u, v)}
$$

and

$$
\Phi(u, v)=\arctan \left\{\frac{\mathcal{I}(u, v)}{\mathcal{R}(u, v)}\right\}
$$

By normalizing every complex number by dividing both real and image parts by $\mathcal{M}(u, v)$ we essentially remove all regular patterns at every scales at once. Note that we don't have to analyze the Fourier image. This normalization works on all frequencies at once and eliminates the regularities. The algorithm of the first stage can now be written (algorithm 1):

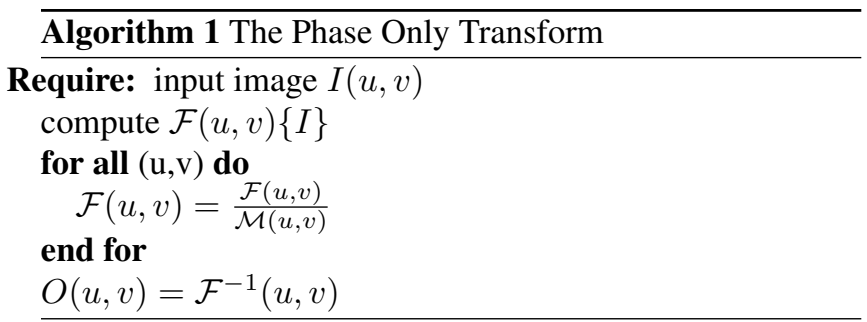

The resulted image is $O(u, v)$. The spectral magnitude of images tends to fall off at high frequencies, the phase-only signal will experience a high-frequency emphasis which will accentuate narrow events without modifying their position. It is reasonable to identify $\frac{1}{\mathcal{M}(u, v)}$ as generally emphasizing high frequencies over low frequencies.

\subsection{Theoretical justification}

We wish to explain why Phase-Only Transform (PHOT) works. For this we want to justify the apparent fact that for texture, the integral excursion of the phase is small compared to that of a defect in the texture.

\subsubsection{Phase of regular texture}

We limit ourselves to $1-\mathrm{D}$, as the discussion extends readily to n-D due to the FT separability, and we carry out the discussion in the continuous domain for simplicity. We need some definitions to start:

Definition 1 (Phase integral excursion) The integral excursion $\mathcal{E}$ of the phase of a real signal is a measure of the range of values that the phase of its FT actually takes. More precisely, let $S(x)$ be a real signal. Let

$$
\mathcal{F}[S](\omega)=\frac{1}{\sqrt{2 \pi}} \int_{-\infty}^{+\infty} S(x) e^{-i \omega x} d x=a_{S}(\omega) e^{i \phi_{S}(\omega)}
$$

be its FT with $\omega$ real belonging to $[0,2 \pi]$. Assuming $\phi_{S}(x)$, the phase of $S$, to be either monotonic or discrete on that domain, we define $\mathcal{E}[S]=\int_{0}^{2 \pi}\left\|\phi_{S}^{-1}(y)\right\| d y$, where $\phi_{S}^{-1}$ is the reciprocal function to $\phi_{S}$.

In essence, the phase integral excursion is the projection on the $y$-axis of the values of $\phi_{S}(\omega)$, the phase of the FT of the signal can take. We limit ourselves to monotonic or discrete phases as this is sufficient for our formalism, and this allows us to define $\phi_{S}^{-1}$ implicitly.

To continue, we need a simple definition of texture.

Definition 2 (regular texture) We define a regular texture as a real signal composed of at most countably infinite linear combinations of the form $S(x)=\cos (a x+b)$ with a and $b$ real.

Such a texture is essentially a slight generalization of a Fourier series. We know Fourier series can represent any bounded periodic signal to an arbitrary degree of precision, which is what we require to represent regular textures.

We have then the following theorem :

\section{Theorem 1 (Integral excursion of a regular texture)}

The integral excursion of any regular texture over a finite domain is zero.

Proof: The FT of $S$ reduces to a superposition of a countable number of Dirac peaks and its phase is such that for all countable $a, \phi_{S}(a)=b$ and zero everywhere else. Therefore $\phi_{S}^{-1}$ is zero almost everywhere, and its Lebesgue measure is zero, and so is the phase integral excursion.

\subsubsection{Phase of a defect}

A defect in a texture can be defined as an abrupt change in its regularity. To study this we model it by a box function.

Definition 3 (Random box function) A random box function $H(a, b)$ is a function which has the following form:

$$
H(a, b)[x]= \begin{cases}0 & \text { if } x<a \\ 1 & \text { if } a \leq x \leq b \\ 0 & \text { if } x>b\end{cases}
$$

We use the following well-known properties of the Fourier transform to derive the expression of the FT of $H(a, b)$.

- Translation invariance : $\mathcal{F}\left[f\left(x-x_{0}\right)\right](\omega)=$ $e^{-i x_{0} \omega} \mathcal{F}[f(x)](\omega)$.

- Scale invariance : $\mathcal{F}[f(a x)](\omega)=\frac{1}{|a|} \mathcal{F}[f(x)]\left(\frac{\omega}{a}\right)$.

- The expression of the centered box function : $H\left(-\frac{1}{2}, \frac{1}{2}\right)=\frac{\operatorname{sinc}\left(\frac{\omega}{2}\right)}{\sqrt{2 \pi}}$ 
The expression of the FT of the random box function is therefore :

$$
\mathcal{F}[H(a, b)](\omega)=e^{-i\left(\frac{a+b}{2}\right) \omega}\left[\frac{\operatorname{sinc}\left(\frac{\omega}{2(b-a)}\right)}{\sqrt{2 \pi}}\right] .
$$

The phase of this FT is simply

$$
\phi_{H(a, b)}[\mathcal{F}[H(a, b)]](\omega)=-\left(\frac{a+b}{2}\right) \omega .
$$

We now have the following theorem :

Theorem 2 (Phase excursion of the random box function) The phase excursion of the random box function is non-zero unless $a+b=0$.

Proof: Ignoring phase wraparound over $2 \pi$, If $a+b \neq 0$, then $\phi_{H(a, b)}$ is monotonic non constant, and so, even including phase wraparound, $\left|\phi_{H(a, b)}^{-1}\right|$ is zero at at most a finite number of points and strictly positive elsewhere. Its integral over any measurable non-empty set is therefore nonzero, and so is the integral phase excursion. We note that since $a$ and $b$ are random, the probability of $a+b=0$ is zero.

Let us assume a regular texture on the one hand, and a regular texture with a defect in the other. Theorem 1 tells us that the former has a FT with a phase composed of negligibly few different values. On the other hand, the latter might be viewed as a linear combination of a regular texture and a random box function with random values for $a$ and $b$. Theorem 2 tells us that the latter FT features a fundamentally different phase composed of uncountably infinite different values with probability one. We now show that the phase-only transform can readily distinguish between these two cases even in the discrete case, as we now illustrate.

\subsection{D examples}

In this section we show a few examples on $1 D$ signals and give some insights about the behavior of the Phase Only Transform. We refer to the PHOT here, as the signal that is transformed back to the spatial domain, after being normalized by the magnitude. As already shown by the $2 \mathrm{D}$ example, most of the information on edges and sharp peaks is contained in the phase. If a signal contains a single peak or edge and a flat region, the phase part of the FFT must be significant, to allow the sum of trigonometric function to be flat. On the other hand, if a signal is constructed of a sum of pure sine or cosine functions of various frequencies with zero or little phase content, the PHOT will be almost zero. This is true not only for signals that are periodic within a finite support. Figure 2 shows such a signal. In Figure 3 we see a sharp peak that requires large phase content. We conclude that signals (not necessarily periodic) that have

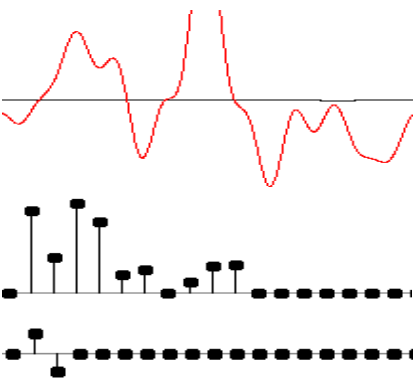

Figure 2. A signal with minor phase content - the PHOT is almost flat. Top: signal (red) and its PHOT (black). Middle: magnitude of frequencies. Bottom: phase of frequencies.

a small phase content would yield a smooth PHOT, while those with large phase content representing a peak or an edge yield a large peak in the PHOT which corresponds to the location of the peak or edge in the input signal. Assuming that a defected signal is composed of sum of sine function of various frequencies and a peak, the result of the PHOT is a collection of peaks in the spatial domain that are localized in the original defect location while the part that is corresponding to the first term is eliminated. Figure 4 shows a small defect (peak), composed with a sine (or cosine) wave. In Figure 5 we show another example on a signal that appears non-periodic due to the limited domain, yet, is composed from a sum of trigonometric functions which are all removed, while the defect remains.

Our model of an input signal is thus composed of two terms, a non-defected term, $A(x)$ which is a sum of sine or cosine functions with relatively small phase content, and a defect term, $B(x)$ which is assumed to be a peak or step edge, thus contains large phase content:

$$
S(x)=A(x)+B(x)
$$

Since the PHOT eliminates the sum of (low phase content) sines, we are left mainly with $B(x)$, as expected from section 3.2. The inverse transform then yields the localization of the defect in the spatial domain.

\subsection{Thresholding using Mahalanobis distance}

In order to be able to use a totally unsupervised method with no learning phase, we have to assume that for each input image the majority of the image pixels are intact (see Section 4). In this case, we can use simple statistics. We use the result of the PHOT as a probability map of a pixel being a defect. As commonly used, we assume a Gaussian distribution and use the Mahalanobis distance. We compute the mean and variance of the distribution from the image obtained by the PHOT. Since we normalize each of the FFT basis when we reconstruct the PHOT image, the global mean and sigma of the image are now both $1 / N$ where $N$ is 


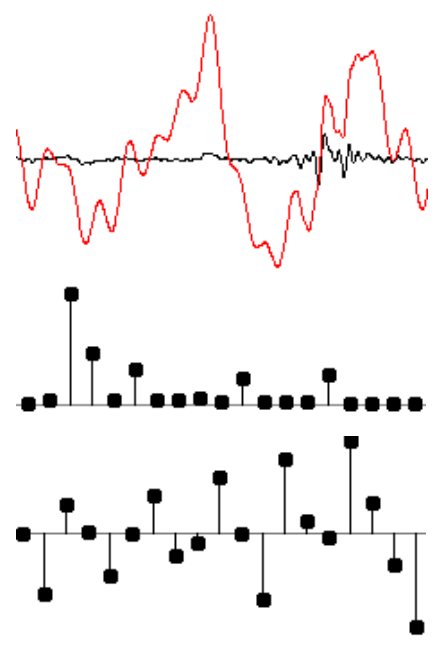

Figure 3. A small defect in a sum of sine curves. Top: signal (red) and its PHOT (black). Middle: magnitude of frequencies. Bottom: phase of frequencies.

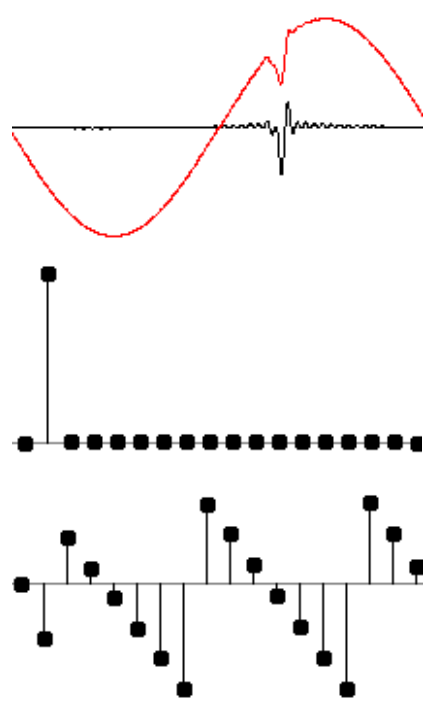

Figure 4. A defect in a single sine curve. Top: signal (red) and its PHOT (black). Middle: magnitude of frequencies. Bottom: phase of frequencies.

the number of pixels. However, since the noise can be significant, we first smooth the PHOT image by a Gaussian filter and only then compute the mean and variance (we have used Gaussian of $\sigma=3.0$ ). The user gives a value in sense of Mahalanobis distance. We threshold the PHOT result such that every pixel with a distance larger than this value is considered as a defected pixel. Figure 6 shows an input image, the PHOT result interpreted as Mahalanobis distance from the mean and the thresholding result using a Mahalanobis distance of 4.0. Of course, once the result of the PHOT is obtained, any other statistical method can be
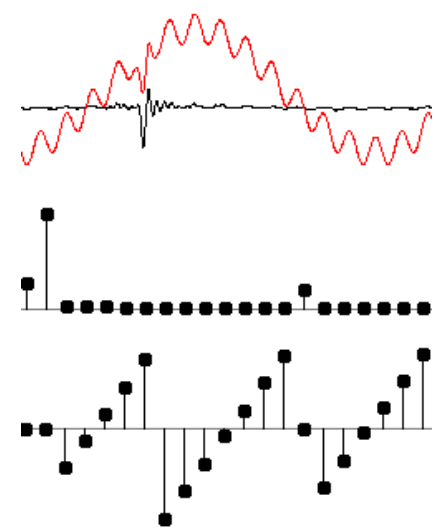

Figure 5. Non periodic (but with little phase) signal and a defect (large phase content). Top: signal (red) and its PHOT (black). Middle: magnitude of frequencies. Bottom: phase of frequencies.

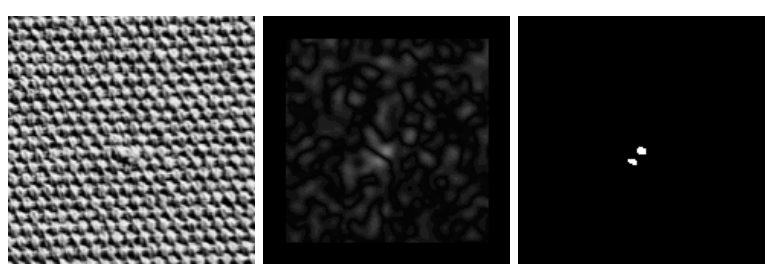

Figure 6. Image in the process of defect detection: left - input image, middle - Mahalanobis distance from the mean (multiply by 30 for visualization), right - thresholding using distance 4.0

also used instead of the normal distribution and the simple Mahalanobis distance.

\section{Characteristics and limitations of the Phase Only Transform}

The most appealing characteristic of the PHOT is that it removes any regularities from the image without the need to identify peaks in the Fourier domain. Only spikes that do not correspond to a sum of trigonometric functions inside the image domain are left. Note that the regularities should not be presented in the entire image. Every large enough regular patterns are removed by the transform by normalizing the resulted complex number by its magnitude. In this sense, our method is different from those that work only on periodic patterns. Figure 7 shows an example of image that has several subpatterns that are regular but the entire image is not. The only parameter in the threshold on the Mahalanobis distance and it is exactly the same in Figures 6 and 7. The result shows that the PHOT has no difficulty in detecting defects in this image. The results look very similar to the human perception of "novel pattern". The entire image is not regular but contains patterns that in some way similarly perceived. We should note here that this can be 

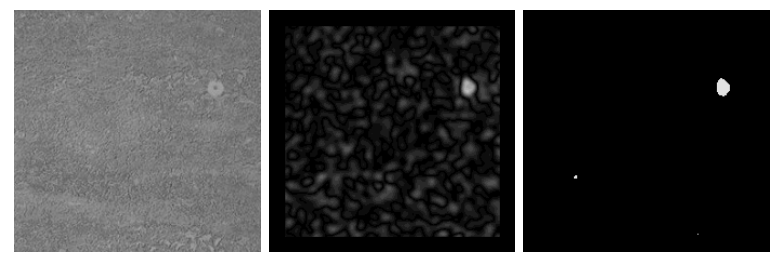

Figure 7. Non regular patterns: left - input image, middle - Mahalanobis distance from the mean (multiply by 30 for visualization), right - thresholding using distance 4.0

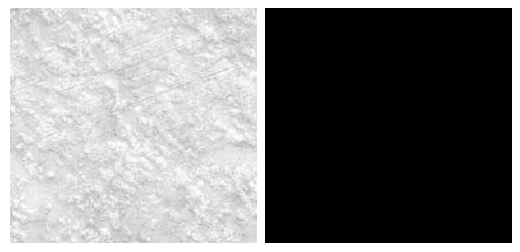

Figure 8. Limitation of 2D transform: Scratches could not be detected as they are $1 \mathrm{D}$ regular.

also considered as a limitation of the method, since large defects can be viewed as regular subpattern, thus might be removed by the PHOT.

As can be expected, if we use 2D FFT on the image, every periodicity or regularity (or homogeneity) is removed by the PHOT. This contains also large defected patterns and $1 \mathrm{D}$ structures. For example, a defect structured as a line or scratch in the image, would not be well detected as can be seen in Figure 8. On the other hand, the same characteristic, can be used (as an advantage) to obtain defect detection on multiple patterns where nothing has to be known by the algorithm in advance ("blind" defect detection). In Figure 9 the results of our algorithm on a image that contains two totally different regularities are shown. It can be observed that the boundary regions between regularities were removed by the PHOT. This means that 1D long defected patterns may not be detected. A way to solve this problem is to apply the PHOT on lines instead on the entire 2D image. This would work however only in a highly regular patterns. We will investigate this direction in the future.

\section{Complexity and real time performance}

In many inspection system that apply defect detection algorithms for quality assurance, the time performance of the algorithm is critical as it might be used in a real manufacturing process. As can be easily concluded from our algorithm, the complexity is $O(n \log n)$ where $n$ is the number of pixels in the input image. This, of course, comes from the DFT that we have to apply. The further processing and statistics is obviously linear with $n$. For very large or continuously inspected patterns, one can apply the algorithm on partial sub-windows without affecting the detection per-

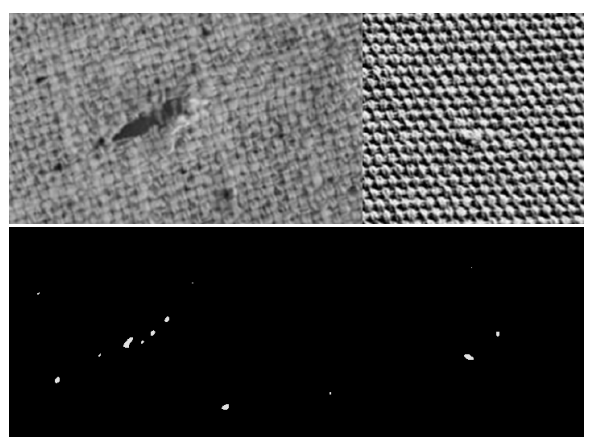

Figure 9. Multiple patterns: top - input image, bottom - thresholding using distance 4.0

formance substantially . It is also very simple to implement the algorithm on parallel machines by decomposing the input. We successfully implemented the algorithm on a GPU (Graphics Processing Unit) with Nvidia CUDA. The FFT is also quite fast in practice and effective parallelization exists using Intel's SSE2 and SSE3 instructions.

\section{Results}

We implemented the algorithm using $\mathrm{C}++$ and Visual Studio. the results on a large set of images are shown in Figures 10. All the results were obtained using the same parameter for thresholding the Mahalanobis distance (4.0). No other parameter is needed for our algorithm. The sensitivity of the algorithm can be changed by the user by altering the Mahalanobis threshold.

\subsection{Multiple sub-patterns and arbitrary patterns}

As already mentioned in Section 4, our method does not require that the entire inspected pattern be regular. It can process many sub-patterns simultaneously. In fact, the PHOT is a detector for novel patterns. It emphasizes patterns that do not appear much in the image. It is worth noting that we do not assume anything about the size of the pattern so it can vary. In Figure 11 an image containing many texture patches of different size and regularities is proceeded and the result (using Mahalanobis threshold 4.0) is shown on the right. The synthetic defect almost invisible by eye in the image is detected since it is novel. Another spike on top of the image is also detected. In Figure 12 a scene that contains a house with textured roof is shown. The image contains textures as well as homogeneous and irregular regions. The synthetic defect as well as the novel pattern of the lamp on the right are well detected.

\subsection{Images with no defects}

We tested our simple adaptive threshold on input images which are texture patches without any defect. The purpose 


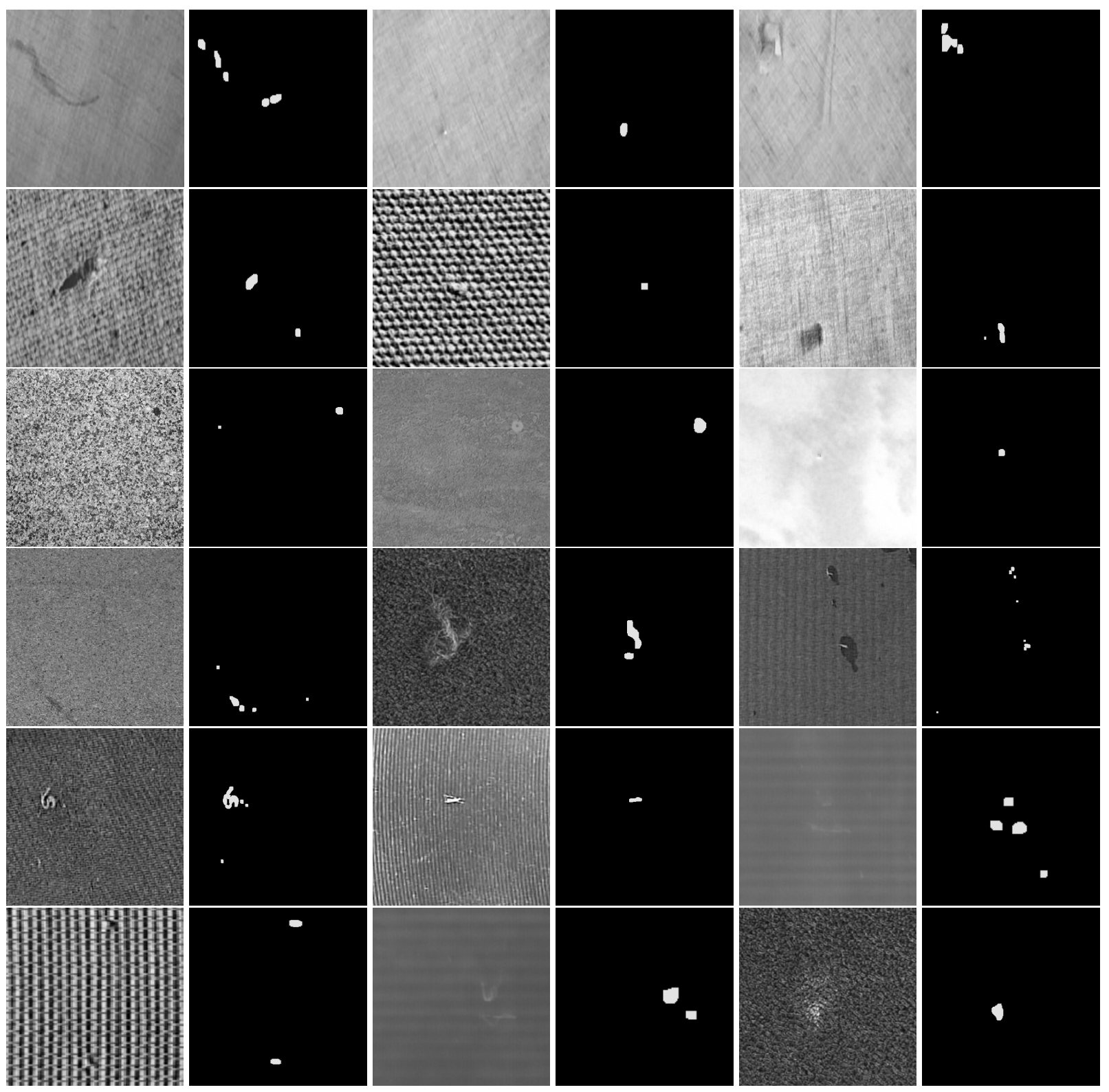

Figure 10. Results on various patterns: in each of the three columns, left - input image, right - results by thresholding using distance 4.0

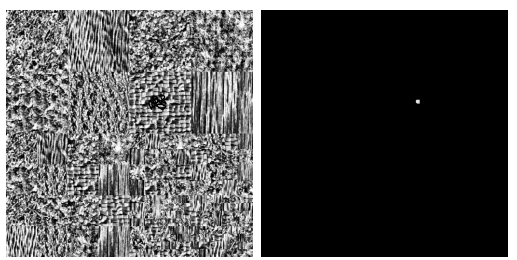

Figure 11. Multiple textures of various size and regularities and a synthetic defect: top - input image, bottom - thresholding using distance 4.0

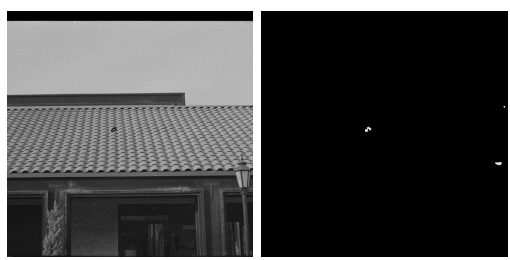

Figure 12. Arbitrary scene with synthetic defect: top - input image, bottom - result

of this test is to verify that the method does not produce false positives. We used exactly the same parameter as in 

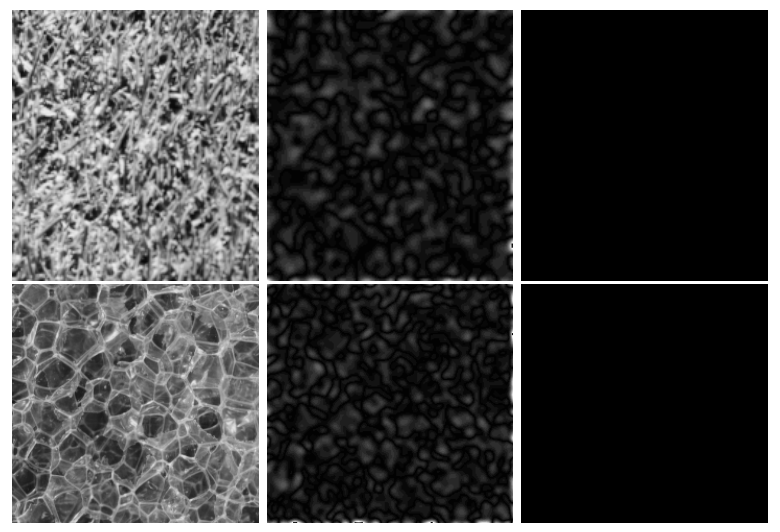

Figure 13. Images with no defects: left - input image, middle PHOT result (multiplied by 30 for visualization), right - result using threshold of 4.0

all other tests, namely, a Mahalanobis distance $=4.0$. In Figure 13 we show two texture patches which are not quite regular (to make the test more difficult), their PHOT results and the output using threshold equal to 4.0. It can be seen that no false positive defects were produced for either inputs. It can be observed in the PHOT result (middle), how the strength of the response is related to the perception of "novelty". Although no pixel exceeds distance 4.0, some regions have larger response correlated to the measure of their regularity.

\section{Conclusions}

A novel method for defect detection on surface patches was presented. The main advantage of the new algorithm is its extreme simplicity (it consists manly of a standard FFT), its generality to work for various pattern without prior knowledge and the fact that it is unsupervised. We gave theoretical justification for a reasonable model. We show results on a large set of inputs and the results are very similar to the perception of defects where no prior information is given. The new algorithm has only one parameter which is the sensitivity of the algorithm. It is an advantage in real inspection systems, where ease of use is important. The algorithm is also fast in practice and can be used in real time systems. Moreover, parallelization of the algorithm can be easily obtained by simply subdividing the input.

\section{References}

[1] A. B. M. Bennamoun and S. Latham. Optimal gabor filters for textile flaw detection. Pattern Recognition, 35:29732991, 2002. 1

[2] C. Chan and G. Pang. Fabric defect detection by fourier analysis. IEEE Transactions on Industry Applications, 36(5):1267-1276, 2000. 2
[3] J. Coggins and A. Jain. A spatial filtering approach to texture analysis. Pattern Recognition Letter, 3:195-203, 1985. 1

[4] M. Comer and E. Delp. Segmentation of textured images using a multiresolution gaussian autoregressive model. IEEE Transactions on Image Processing, 8(3):408-420, 1999. 1

[5] F. DAstous and M. Jernigan. Texture discrimination based on detailed measures of the power spectrum. International Conference on Pattern Recognition, 3:83-86, 1984. 2

[6] W. Freeman. The fractal geometry of nature. 1983. 1

[7] R. Haralick. Statistical and structural approaches to texture. Proceedings of the IEEE, 67(5):786-804, 1979. 1

[8] C. D. Kuglin and D. C. Hines. The phase correlation image alignment method. IEEE Int. Conf. on Cybernetics and Society, pages 163-165, 1975. 2

[9] A. Kumar and G. Pang. Fabric defect segmentation using multichannel blob detectors. Optical Engineering, 39(12):3176-3190, 2000. 1

[10] S. Li. Markov random filed modeling in image analysis. 2001. Springer. 1

[11] L. M. G. C. Z. W. X. Luo and H. Lu. Phase-only transform based shaping for error concealment coding. MobiMedia, 11, 2006. 2

[12] J. E. R. N. M. Millan and J. Pladellorens. Detection of local defects in textile webs using gabor filters. Optical Engineering, 37(8):2297-2307, 1998. 1

[13] A. M. M. Mirmehdi and B. Thomas. Restructured eigenfilter matching for novelty detection in random textures. British Machine Vision Conference, pages 637-646, 2004. 1

[14] M.Markou and S. Singh. Novelty detection: A review part 1: Statistical approaches. Signal Processing, 83:2481-2497, 2003. 1

[15] M.Markou and S. Singh. Novelty detection: A review part 2: Neural network based approaches. Signal Processing, 83:2499-2521, 2003. 1

[16] F. V. R. Nevatia and K. Price. Structural analysis of natural textures. IEEE Transactions on Pattern Analysis and Machine Intelligence, 8:76-89, 1986. 1

[17] A. V. Oppenheim and J. S. Lim. The importance of phase in signals. IEEE, Proceedings, 69:529-541, 1981. 2

[18] T. Reed and J. Buf. A review of recent texture segmentation and feature extraction techniques. Computer Vision, Image Processing and Graphics, 57(3):359-372, 1993. 1

[19] D. Tsai and C. Heish. Automated surface inspection for directional textures. Image and Vision Computing, 18(1):4962, 1999. 2

[20] M. Tuceryan and A. Jain. Texture analysis. In Handbook of Pattern Recognition and Computer Vision, pages 235-276, 1998. chapter 2, World Scientific, 1998. 1

[21] X. Xie. A review of recent advances in surface defect detection using texture analysis techniques. Electronic Letters on Computer Vision and Image Analysis, 7:1-22, 2008. 1

[22] X. Xie and M. Mirmehdi. Texem: Texture exemplars for defect detection on random textured surfaces. IEEE Transactions on Pattern Analysis and Machine Intelligence, 29(8):1454-1464, 2007. 1 